\title{
1977-2006: Trente années de mesures des températures de l'eau dans le Bassin du Rhône
}

\section{7-2006 : Thirty years of water temperature measurements in the Rhône Basin}

\author{
A. Poirel, F. Lauters, B. Desaint \\ EDF/DTG, 21 av. de l'Europe, BP 41, 38040 Grenoble Cedex, France \\ e-mail : alain.poirel@edf.fr
}

Résumé - La température de l'eau du Rhône et de ses affluents est mesurée au pas de temps horaire depuis 1977 sur une quinzaine de stations. La constitution de ces chroniques a nécessité un effort constant de métrologie, de validation et de critique sur plus de trente années pour obtenir des données fiables. L'analyse de ces données permet de définir le régime thermique du Rhône et de mettre en évidence d'importantes augmentations thermiques (de +1 à $+2{ }^{\circ} \mathrm{C}$. sur les moyennes annuelles). Ces augmentations sont plus élevées sur le Rhône aval et sur ses affluents chauds. L'effet est plus marqué au printemps et en été, excepté sur les stations soumises à un régime hydrologique nivo-glaciaire.

Mots clés - température de l'eau, série chronologique, Rhône

Abstract - Water temperature of the Rhone and its tributaries is measured hourly since 1977 on about fifteen stations. The installation and maintenance of these stations to get reliable data required a constant effort in metrology and data validation over thirty years. The analysis of these time series data characterizes the thermal regime of the Rhone and highlight significant increases in water temperature $\left(+1\right.$ to $+2^{\circ} \mathrm{C}$ on annual means). These increases are higher downstream on the Rhone and some warmer tributaries. The effect is more pronounced in spring and summer, except on sites subject to a glacial or nival hydrological regime.

Key words - water temperature, time series, Rhône River

\section{INTRODUCTION}

Depuis les années 1970, la température de l'eau des fleuves a été mesurée par EDF dans le cadre du programme thermique classique et nucléaire, que ce soit pour l'étude de la source froide avant construction d'un site ou pour contrôler la température en amont du site ensuite.

Si les séries chronologiques sont assez disparates avant 1975, la fin de l'année 1976 voit la mise en service de nouvelles stations sous 
l'effet de la canicule estivale, stations qui ont été exploitées sur plus de trente ans grâce à la clairvoyance de quelques gestionnaires de réseau. À partir de cette date, on dispose donc de données horaires relativement fiables sur de nombreux points de mesure en amont des sites de production électrique nucléaires (CNPE).

L'étude globale des températures de l'eau du Rhône et de ses affluents, à l'instigation de l'Administration et pilotée par la DRIRE Rhône-Alpes, a été décidée en 1999, sous forme de trois phases complémentaires : 1/quelle est la température du Rhône, 2/ quels sont les facteurs explicatifs en particulier, quelle est la part relative due aux échauffements des Centrales Thermiques (classiques et nucléaires) et $3 /$ quels sont les effets biologiques de ces températures.

Cet article fait la synthèse de la première phase qui vise à préciser le régime thermique du Rhône sur 19772003, ainsi que les tendances observées. Les résultats obtenus de 2004 à 2006 ont été pris en compte pour compléter les données et atteindre ainsi trente années de mesures.

Cette première phase d'étude a pris le parti de rester factuelle, sans chercher à expliquer les températures mesurées, objet de la seconde phase. Ce point peut apparaître frustrant à la lecture mais l'objectif premier était bien de constituer des longues séries de données complètes, validées et critiquées, base obligatoire de toute analyse plus détaillée. Cela constitue en soit un travail très important et parfois fastidieux compte tenu des volumes d'informations à traiter et des longues périodes sur lesquelles elles s'étendent.

\section{PRÉSENTATION DES SITES DE MESURE}

Le bassin du Rhône est équipé de plusieurs stations de mesures de la température de l'eau situées pour la plupart en amont immédiat des sites thermiques mais aussi aux principaux nœuds du réseau hydrographique (Fig. 1). Compte tenu de la morphologie du fleuve, le mélange des eaux est souvent assez médiocre que ce soit au niveau des confluences ou des rejets. C'est pourquoi, des stations en aval proche des rejets thermiques, n'ont pas été prises en compte.

Les séries de données retenues sont donc celles qui présentent à la fois une portée temporelle intéressante, mais aussi celles qui sont représentatives du fleuve et non d'un effet local :

- Pougny (POU) : première station à $25 \mathrm{~km}$ en aval du Léman. La station est située à l'aval immédiat du pont de Pougny qui marque la frontière Franco-Suisse.

- Creys-Malville (CRE) : station située au niveau du CNPE de CreysMalville. La station est sur un ponton flottant situé à la prise d'eau de la centrale, en amont du rejet.

- Bugey (BUG) : station située au niveau du CNPE de Bugey. La station est dans l'enceinte du CNPE, au niveau de la prise d'eau de la centrale, en amont du rejet.

- Jons (JON) : station située en aval de la confluence Ain-Rhône, au niveau du barrage de Jons (amont de Lyon). La station est située côté Rhône court-circuité (canal de Jonage). L'homogénéité thermique 
de cette station est parfois douteuse mais sa situation en amont de la Saône est intéressante.

- Loire sur Rhône (LSR) : station située en aval de Lyon et de la confluence Rhône-Saône, en amont immédiat de Vienne, au niveau de la prise d'eau de l'ancienne centrale thermique de Loire sur Rhône, fortement redondante avec la station suivante de Saint Alban.

- Saint Alban (SAL) : station située en aval de Vienne en rive droite au pont de Chavannay jusqu'en 1998 et en rive gauche, en entrée de la prise d'eau du CNPE de Saint Alban depuis 1997.

- Seuil de Peyraud (SPE) : station située en aval du CNPE de Saint Alban, dans le tronçon courtcircuité par l'usine hydroélectrique de Sablons (Péage de Roussillon). Cette série débute en 1985 seulement mais constitue une référence des Vieux Rhône.

- Roche de Glun (RGL) : station en amont de l'Isère, située entre Tournon et Valence en aval du pont de Roche de Glun. Cette série débute en 1995 seulement.

- Soyons (SOY) : station située en aval de l'Isère, à proximité de Valence, environ $500 \mathrm{~m}$ en aval du port de Valence.

- Cruas (CRU) : station située en aval de la confluence Rhône-Drôme et en amont de Montélimar. La station est en aval immédiat du barrage de Logis Neuf sur le canal usinier.

- Tricastin (TRI) : station située au niveau de Pierrelatte. La station est sur le canal de Donzère en amont immédiat du CNPE de Tricastin.

- Aramon (ARA) : station située en aval d'Avignon et de la confluence
Durance-Rhône, au niveau du CPT d'Aramon (amont rejet).

Trois stations sont sur les principaux affluents :

- Pont de Chazey (AIN) : station située entre le barrage d'Allement et la confluence Ain-Rhône. La station est immédiatement en aval du pont de Chazey.

- Couzon (SAO) : station située entre Villefranche et la confluence Saône-Rhône en aval de Neuville/ Saône. La station est placée en aval immédiat du barrage écluse de Couzon. Cette station a été déplacée à Macon en 1998.

- Beaumont Monteux (ISE) : la station est située en amont de la confluence Isère/Rhône, à l'aval immédiat du barrage de Beaumont Monteux.

À ces données produites par EdF ont été ajoutées les données Fédérales Suisses de l'OFEG

- SCX : Rhône à l'amont du Léman (Porte du Scex) sur la période 1997-2006.

- ARV : l'Arve au Bout du Monde sur la période 1997-2006.

- LEM : le Rhône à l'aval du Léman et l'amont de l'Arve (Rhône à Halle de l'lle) sur la période 1977-2006.

Les données récentes de la Durance ont été ajoutées mais la chronique mesurée est trop courte aujourd'hui pour permettre une analyse basée sur des mesures.

- DUR : série reconstituée par modèle de la Durance à Bonpas sur la période 1977-2006. 


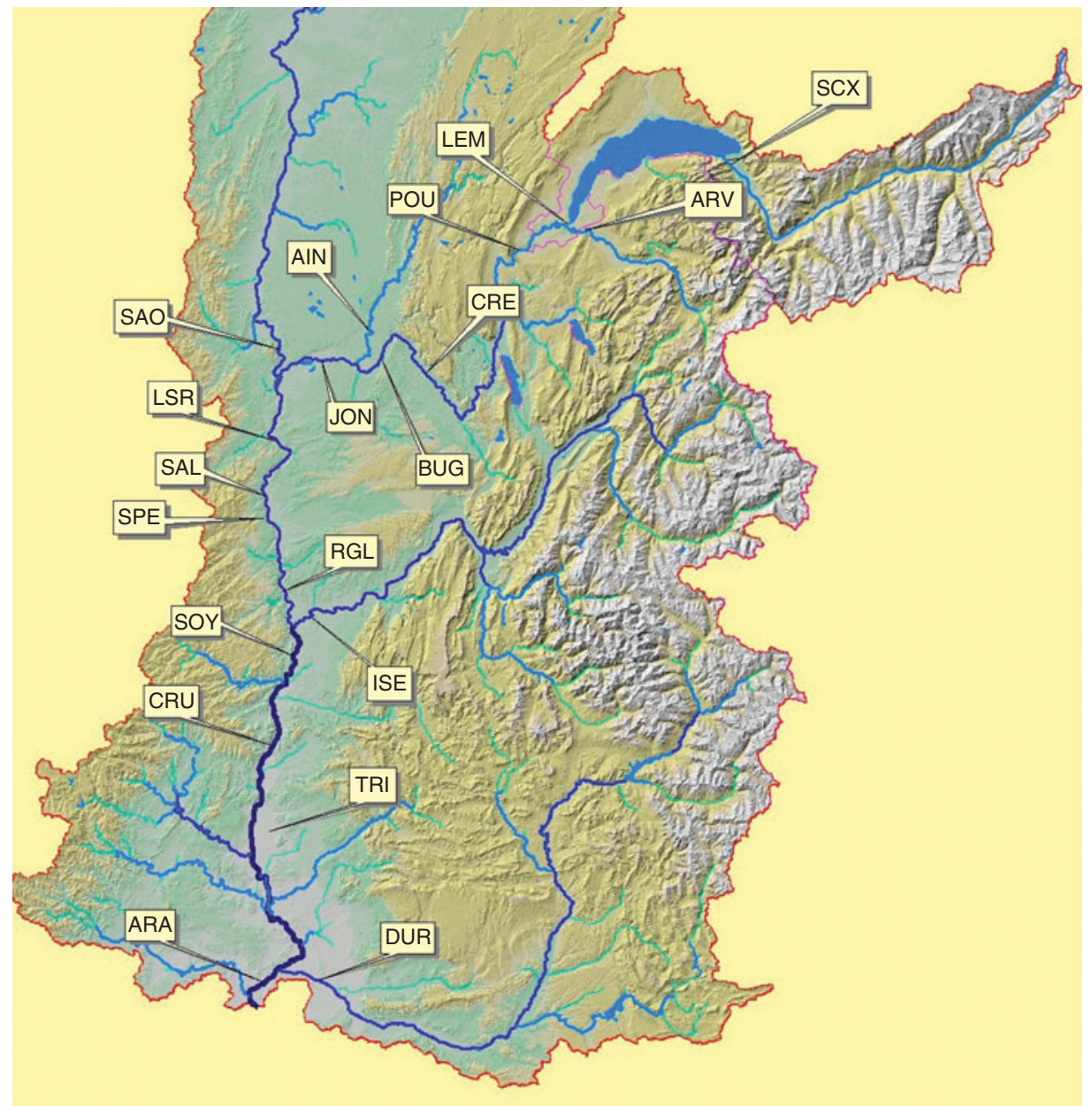

Fig. 1. Bassin du Rhône et localisation des points de mesure.

Fig. 1. Basin overview and location of sampling stations.

\section{ACQUISITION, VALIDATION ET CRITIQUE DES DONNÉES}

\subsection{Description technique des chaînes de mesure}

Les chaînes de mesure de la température de l'eau utilisées par EDF sur le Rhône sont constituées de quatre composants : un capteur contenant le gaz ou la résistance de platine qui varie en fonction de la température interne du capteur, un convertisseur thermométrique, un enregistreur et une alimentation. Cinq générations différentes de chaînes de mesure des 
Tableau I. Générations successives de capteurs et centrales d'acquisition.

Table I. Successive generations of temperature sensors and data acquisition systems.

\begin{tabular}{|c|c|c|c|c|c|}
\hline & $\begin{array}{l}\text { Chaîne de } \\
\text { génération } 1\end{array}$ & $\begin{array}{l}\text { Chaîne de } \\
\text { génération } 2\end{array}$ & $\begin{array}{l}\text { Chaîne de } \\
\text { génération } 3\end{array}$ & $\begin{array}{l}\text { Chaîne de } \\
\text { génération } 4\end{array}$ & $\begin{array}{l}\text { Chaîne de } \\
\text { génération } 5\end{array}$ \\
\hline Capteur & $\begin{array}{l}\text { Sonde à gaz } \\
\text { SIAPE ou } \\
\text { RICHARD }\end{array}$ & $\begin{array}{l}\text { sonde platine } \\
\text { PT100 1/5DIN } \\
\text { classe A ( } 3 \text { fils) } \\
\text { Pyro.contrôle ou } \\
\text { Engelhard }\end{array}$ & $\begin{array}{l}\text { sonde platine } \\
\text { PT100 1/10DIN } \\
\text { classe A (4 fils) } \\
\text { Pyro.contrôle ou } \\
\text { Engelhard }\end{array}$ & $\begin{array}{l}\text { sonde platine } \\
\text { PT100 1/10DIN } \\
\text { classe A ( } 4 \text { fils) } \\
\text { Pyro.contrôle ou } \\
\text { Engelhard }\end{array}$ & $\begin{array}{l}\text { sonde platine } \\
\text { PT100 1/10DIN } \\
\text { classe A ( } 4 \text { fils) } \\
\text { Pyro.contrôle ou } \\
\text { Engelhard }\end{array}$ \\
\hline $\begin{array}{l}\text { Convertisseur } \\
\text { Thermomètre }\end{array}$ & $\begin{array}{l}\text { Thermographe } \\
\text { SIAPE ou } \\
\text { RICHARD }\end{array}$ & $\begin{array}{l}\text { Thermomètre num. } \\
\text { "chaîne B" DTG } \\
\text { Gamme } 0+40^{\circ} \mathrm{C}\end{array}$ & $\begin{array}{l}\text { Carte BIVOIE } \\
\text { DTG } \\
\text { Gamme }-20 \\
+50^{\circ} \mathrm{C}\end{array}$ & $\begin{array}{l}\text { SAB } 600 \mathrm{HDL} \\
\text { CR2M } \\
\text { Gamme }-20 \\
+50^{\circ} \mathrm{C}\end{array}$ & Rosemound \\
\hline $\begin{array}{l}\text { Enregistreur } \\
\text { (modèle } \\
\text { marque) }\end{array}$ & $\begin{array}{l}\text { Enregistreur } \\
\text { papier inclus }\end{array}$ & $\begin{array}{l}\text { Type : Enregistreur } \\
\text { magnétique à K7 } \\
\text { EMK10 ou EMAC } \\
\text { SCHLUMBERGER } \\
\text { ou AUTEG }\end{array}$ & $\begin{array}{l}\text { PA Monovoie } \\
\text { Centralp }\end{array}$ & $\begin{array}{l}\text { SAB } 600 \mathrm{HDL} \\
\text { CR2M } \\
\text { Gamme }-20 \\
+50^{\circ} \mathrm{C}\end{array}$ & $\begin{array}{l}\text { LogoSens } \\
\text { OTT } \\
\text { Gamme }-20 \\
+50^{\circ} \mathrm{C}\end{array}$ \\
\hline $\begin{array}{l}\text { Alimentation } \\
\text { (type } \\
\text { autonomie) }\end{array}$ & $\begin{array}{l}\text { Horlogerie } \\
\text { mécanique } \\
\text { Autonomie : } 1 \\
\text { semaine (papier) }\end{array}$ & $\begin{array}{l}\text { thermomètre : pile } \\
\text { R20x4 = 6V } \\
\text { enregistreur : pile } \\
\text { R20x4 = } 6 \mathrm{~V} \\
\text { autonomie : } 1 \text { an }\end{array}$ & $\begin{array}{l}\text { carte BIVOIE : } \\
\text { pile Li } 6 \mathrm{~V} \\
\text { carte PA : pile } \\
\text { R20x10 = 15V } \\
\text { autonomie : } 1 \mathrm{an}\end{array}$ & $\begin{array}{l}\text { CR2M : } \\
\text { pile R20x4 = 6v } \\
\text { autonomie : 1an }\end{array}$ & $\begin{array}{l}\text { Batterie } 12 \mathrm{~V} \\
\text { réalimentée } \\
\text { Alimentation } \\
\text { Texup batterie } \\
\text { autonomie > 1an }\end{array}$ \\
\hline $\begin{array}{l}\text { Précision de la } \\
\text { chaîne de } \\
\text { température }\end{array}$ & $\begin{array}{l}\text { chaîne complète : } \\
\pm 0,5^{\circ} \mathrm{C}\end{array}$ & $\begin{array}{l}\text { capteur: } \\
\pm 0,3^{\circ} \mathrm{C} \\
\text { thermomètre: } \\
\pm 0,1^{\circ} \mathrm{C}\end{array}$ & $\begin{array}{l}\text { capteur: } \\
\pm 0,15^{\circ} \mathrm{C} \\
\text { thermomètre : } \\
\pm 0,1^{\circ} \mathrm{C}\end{array}$ & $\begin{array}{l}\text { capteur : } \\
\pm 0,15^{\circ} \mathrm{C} \\
\mathrm{CR} 2 \mathrm{M}: \\
\pm 0,1^{\circ} \mathrm{C}\end{array}$ & $\begin{array}{l}\text { capteur: } \\
\pm 0,15^{\circ} \mathrm{C} \\
\pm \mathrm{OTT}: 0,1^{\circ} \mathrm{C}\end{array}$ \\
\hline Période & 1977-1985 & $1985-1990$ & 1990-1998 & 1998-2002 & depuis 2002 \\
\hline
\end{tabular}

températures de l'eau se sont succédées.

Elles se différencient soit par des améliorations métrologiques, soit par des améliorations sur la fiabilité et la maintenance notamment avec les systèmes d'acquisition en temps réel (Tab. I).

\subsection{Méthodologie du choix d'un point de mesure}

Les sections de mesures ont toujours été étudiées en préalable à l'installation par des profils thermiques à bas débit pour en vérifier l'homogénéité. Le capteur est positionné à plus d'un mètre de profondeur pour éviter les effets du rayonnement solaire direct, dans une zone courante mais néanmoins protégée des corps flottants.

L'implantation des chaînes de mesure est variable d'une station à l'autre en fonction de la configuration du terrain, mais on retrouve un schéma type d'implantation :

- Boîtier ou armoire en rive contenant le convertisseur thermométrique, l'enregistreur et l'alimentation.

- Gaine de protection du câble de liaison entre le transmetteur et le capteur prolongée par un tube inox avec la crépine contenant le capteur. La gaine de protection chemine en rive puis au fond de la 
rivière ou sur un support artificiel (mur...). Le tube inox avec crépine est fixé sur un support (fond de rivière ou support artificiel comme un mur, une bouée...).

\subsection{Gestion des capteurs et des chaînes de mesures}

La gestion des chaînes de mesure de température a évolué en même temps que le matériel de mesure. Le passage à la télétransmission des données dans les années 1990 a permis une diminution des déplacements sur site pour la collecte des données ainsi qu'une diminution de la perte de données sur les incidents grâce à la détection plus précoce des pannes.

L'entretien à effectuer sur les chaînes de mesure est pratiquement nul depuis la mise en service des sondes de platines avec un branchement à « 4 fils » qui limite la dérive des capteurs.

Initialement, ils se limitaient, lors de visite sur site, à une vérification des différents modules de la chaîne de mesure, au changement des piles et à une mesure comparative avec un thermomètre de précision.

Sur les stations sujettes à l'envasement, des injections d'eau par pompe dans le tube permettent de maintenir la crépine en eau courante.

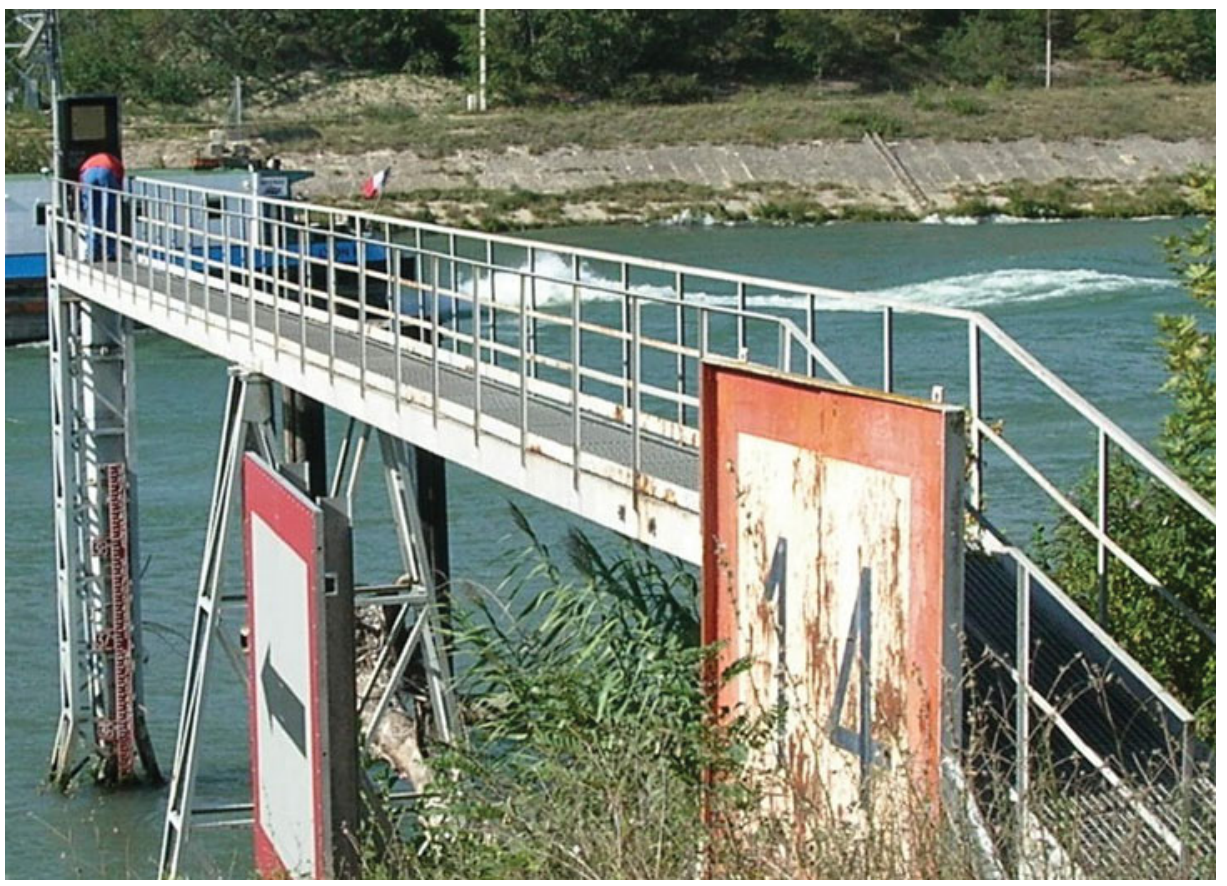

Photo 1. Station de mesure de la température de l'eau sur le Rhône chenalisé.

Photo 1. Water temperature sampling site on the channelized Rhône. 
Depuis 2003, des étalonnages sur sites avec un four thermostaté de précision sont réalisés, de même que des vérifications complètes des transmetteurs et afficheurs. La procédure a été mise au point avec le Laboratoire National d'Étalonnage. Elle a permis de mettre en évidence la difficulté à obtenir in situ une précision inférieure à $0,2-0,3^{\circ} \mathrm{C}$ entre la mesure du capteur et l'enregistrement dans une base de données. C'est finalement la valeur de $0,3^{\circ} \mathrm{C}$ qui a été retenue comme précision raisonnable permettant de déclarer la chaîne de mesure conforme.

\subsection{Réflexions sur la notion de précision et de représentativité des mesures}

La température étant un paramètre fondamental pour de multiples applications, de nombreux efforts ont été consentis pour disposer de mesures de plus en plus précises. Certains constructeurs affichent aujourd'hui une précision de $0,1^{\circ} \mathrm{C}$ voire de $0,01^{\circ} \mathrm{C}$ sur le capteur luimême... Si cette précision est accessible en laboratoire, elle semble beaucoup plus délicate à vérifier pour des mesures in situ et en continu : influence de la température de l'air et de l'humidité sur l'électronique, modification progressive des résistances des connecteurs, décalage liés aux transformations entre signal analogique et signal numérique induisant de nouvelles incertitudes, sans oublier un possible décalage temporel des mesures par dérive du capteur ou par moyennage, etc.

Des parades métrologiques ont été mises en œuvre pour remédier à ces défauts : passage des sondes à 3 fils vers des sondes à 4 fils moins sensibles aux résistances de fils, fiabilisation des composants électroniques dans la gamme des températures externes.

Avec 30 années de mesures et 5 générations de chaînes de mesure, il subsiste inévitablement des hétérogénéités sur la précision des données.

Le second élément de réflexion à prendre en considération est la notion de représentativité spatiale et temporelle de la mesure : un capteur ne mesurant jamais que sa propre température, encore fautil qu'il soit installé dans un milieu homogène, pas influencé par un élément extérieur (tube de fixation par exemple) et que son inertie ne soit pas trop élevée. Malheureusement, il existe fréquemment une hétérogénéité plus ou moins marquée dans les températures de l'eau d'une

rivière selon qu'on se positionne en bordure, en surface, sur le fond, dans le courant principal ou dans une zone d'eau morte, ...

Le choix fait dans l'implantation des capteurs revêt donc une grande importance. Le réseau des thermographes installés sur le Rhône a été conçu pour être représentatif des flux.

Les capteurs ont été installés dans un écoulement notable, plutôt entre 1 et $2 \mathrm{~m}$ de profondeur pour les secteurs profonds du Rhône et suffisamment éloignés de la berge lorsque cela était possible. De ce fait, à une confluence ou après un rejet, on peut additionner les températures proportionnellement aux débits pour trouver une température aval " après mélange calculée ».

Sur le transect de la figure 2 (pour les conditions de débit et 


\section{PROFIL THERMIQUE - TRICASTIN - DROME}

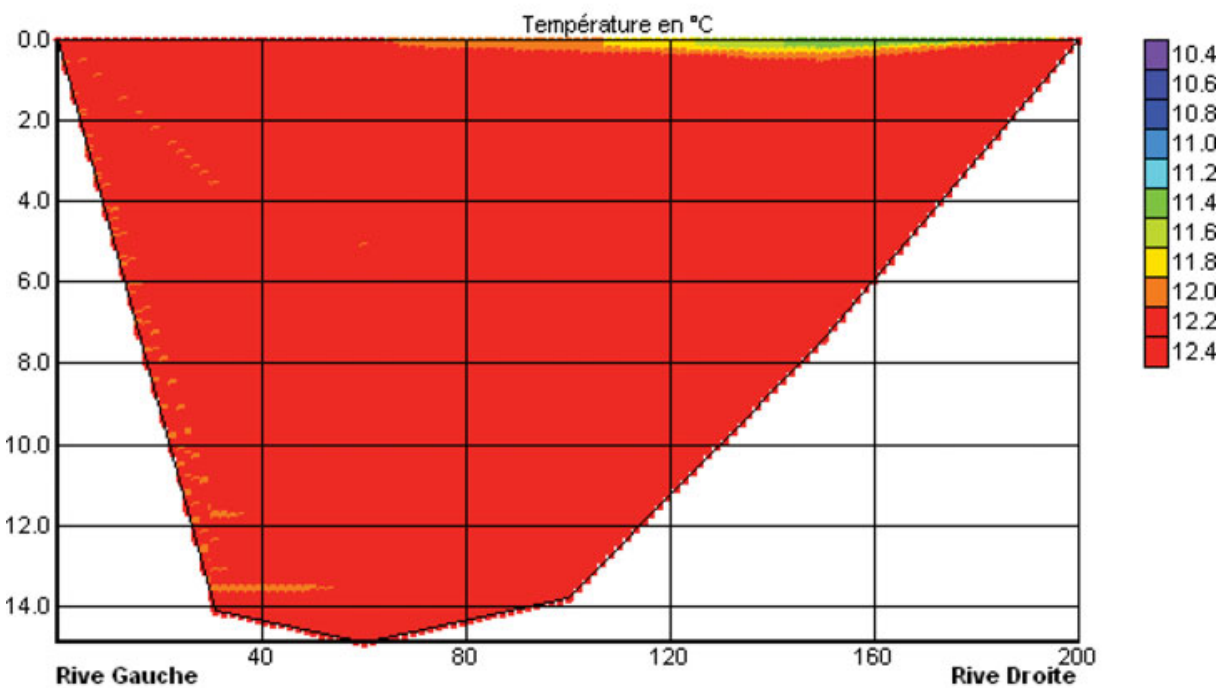

Attention échelle des profondeurs exagérée d'un facteur 8.

Fig. 2. Profil thermique sur le Rhône.

Fig. 2. Water temperature transect on the Rhône river.

météorologiques lors de la mesure), la température est homogène à l'exception de la surface en rive droite plus froide d'un degré environ. À noter que la représentation classique des transects fausse largement la perception des profondeurs par rapport aux hauteurs.

Temporellement, une mesure a été conservée à chaque heure ronde depuis 1977. Les chaînes de mesures ne moyennent donc pas les valeurs autrement que par l'inertie propre du capteur.

Ces mesures sont ensuite agrégées en moyenne journalière, mensuelle, annuelle en abandonnant la logique des flux puisqu'il s'agit de moyennes simples non pondérées par les débits cette fois (i.e. la température moyenne annuelle multipliée par le débit moyen annuel est significativement différente de la température du volume d'eau qui s'écoule durant l'année....).

$\mathrm{Au}$ vu des différents points précédents, il n'apparaît aujourd'hui pas fondamental de mener une course à la précision des capteurs alors que tant d'autres considérations entrent en jeu. Un des progrès à réaliser dans ce domaine consistera à passer progressivement vers la prise en compte des hétérogénéités (Ebersole et al., 2003) pour aboutir à une ou probablement des 
températures biologiquement représentatives.

Cela passera probablement

- par des acquisitions complémentaires sur des habitats préférentiels (Westoff et al., 2007) puis par l'analyse des écarts à la série représentative des flux,

- par une meilleure définition de l'information thermique recherchée en fonction de la sensibilité des organismes (Beitinger \& Bennett, 2000 ; Eaton, 1995) et donc par la définition de métriques pertinentes (Grenouillet et al., 2001; Daufresne \& Boët, 2007) à mettre en regard des mesures biologiques temporellement beaucoup plus rares.

\subsection{Principes de la validation et de la critique des données}

Les mesures brutes sont datées, ce qui leur confère le statut de données brutes. Au début archivées sur papier puis ensuite automatiquement via des centrales d'acquisition, elles sont transférées sur Base de Données avec des identifiants de référence spatiale (un numéro de station) et temporelle (une date et heure dans un système prédéfini).

II existe malheureusement un certain nombre de données aberrantes ou fortement douteuses pour une multitude de raisons : mise à l'air du capteur pour étalonnage ou en étiage sévère, défaut électronique, mauvaise gestion informatique ou mauvaise datation (Moatar, 1997).

Pour détecter et éliminer ces données aberrantes, deux filtres successifs sont mis en œuvre :

- la validation des données consiste à éliminer les données pour lesquelles on a une raison identifiée de penser qu'elles ne représentent pas le paramètre mesuré. Typiquement, des valeurs en dehors de la gamme 0 à $100^{\circ} \mathrm{C}$, des cycles journaliers identiques à ceux observés dans l'air, ... constituent des données à éliminer de la série. Par extension on réalise également l'ébarbage des chroniques en enlevant des pics anormaux et ponctuels, et on complète les manques de données sous réserve qu'ils restent inférieurs à 8 heures durant la journée. À l'issue de ce traitement les données sont réputées validées : on ne peut alors pas exclure qu'un capteur fonctionnant dans des conditions normales ait fourni les valeurs annoncées.

- La critique est constituée d'une ou plusieurs étapes qui visent à obtenir, au final, une série la plus complète et la plus probable possible. Les dérives éventuelles sont recherchées et corrigées (comparaison des valeurs manuelles et automatiques). Les valeurs manquantes peuvent être reconstituées, au pas de temps journalier seulement, soit à partir de stations proches, soit à partir de modèles. Par contre, contrairement aux pratiques hydrométéorologiques classiques, l'utilisation des méthodes de résidus cumulés ou assimilées pour homogénéiser les séries, n'est guère pertinente car des effets réels peuvent conduire à modifier la répartition des écarts entre séries : modification d'un rejet thermique, 
création d'un aménagement hydraulique en amont, changement dans l'exploitation hydraulique du fleuve ou d'un affluent, modification des débits dans les tronçons court-circuités...

\section{ANALYSE DES TEMPÉRATURES DU RHÔNE SUR LA PÉRIODE 1977-2006}

\subsection{Bilan des données disponibles}

Les taux de données horaires disponibles sur les différentes stations avoisinent $98 \%$ sur la période 19771990 et dépassent ensuite 99 \% sur les stations situées en amont des CNPE, là où les mesures ont été doublées. À Bugey amont, par exemple, on compte 258967 mesures horaires présentes pour 4001 valeurs absentes sur la période 1977-2006, et il ne manque que 8 mesures depuis 1984...

Les moyennes journalières calculées et archivées à l'issue de chaque année ont été analysées graphiquement pour vérifier la cohérence d'un travail de validation qui s'est étalé sur trente ans et dans un cadre d'exigences variables...

- Quelques données journalières jugées douteuses et initialement assez mal reconstituées ont été revues (manque de recul statistique sur les données à l'époque). Ces corrections représentent moins de 250 jours sur les 100000 données journalières utilisées.

- Quelques dérives de capteurs ont également été détectées surtout les premières années où les sondes utilisées étaient à « 3 fils » et donc sujettes à des résistances parasites de câble ou de connecteur (moins de 500 jours).

- Quelques biais sur des périodes longues ont été corrigés à partir des valeurs manuelles, notamment pour un type de transmetteur pouvant induire un léger offset (environ $10 \%$ des valeurs corrigées de 0,1 à $0,2^{\circ} \mathrm{C}$ en général, de $0,4^{\circ} \mathrm{C}$ au maximum).

Au final, sur les 100000 valeurs journalières utilisées dans ce travail, moins de 1000 ont été corrigées notablement et 10000 ont été très légèrement modifiées.

\subsection{Analyse des évolutions longitudinales}

L'évolution longitudinale des températures de l'eau est analysée au travers de statistiques (Tab. II) traduisant soit des valeurs «habituelles »moyenne, médiane, gamme 25-75\% des valeur -, soit des valeurs élevées - quantiles $90 \%, 95 \%$, $99 \%$, température moyenne du mois le plus chaud, température maximale annuelle -. Quelle que soit la statistique utilisée, dans un cas comme dans l'autre, les résultats sont assez comparables en termes d'interprétation.

Les figures $3 a$ et $3 b$ donnent une idée de la répartition des températures couramment observées (moyennes, quantiles $10,25,75$ et $90 \%$ ) sur les différents points de mesures et ce pour deux périodes de référence.

On observe un gradient général amont-aval à la fois Nord-Sud et altitudinal, fortement marqué par l'influence du Léman qui augmente la température moyenne du Rhône de plus de 
Tableau II. Principales caractéristiques statistiques des séries de données.

Table II. Main statistics of time series data.

\begin{tabular}{lrrrrccc}
\hline Station & Années & Moyenne & Médiane & $\begin{array}{c}\text { Écart } \\
\text { type }\end{array}$ & quantile 95 \% & $\begin{array}{c}\text { quantile 99 \% } \\
\text { Moy. mensuelle } \\
\text { maximale }\end{array}$ \\
\hline SCX & $1997-06$ & 7,36 & 7,90 & 2,31 & 10,50 & 11,20 & 10,42 \\
LEM & $1977-06$ & 11,83 & 10,50 & 5,56 & 21,70 & 24,00 & 24,88 \\
ARV & $1997-06$ & 8,38 & 9,10 & 3,13 & 12,30 & 13,00 & 12,13 \\
POU & $1977-06$ & 11,04 & 10,01 & 4,98 & 19,80 & 21,70 & 21,70 \\
CRE & $1977-06$ & 11,70 & 10,80 & 5,43 & 20,90 & 22,75 & 23,49 \\
BUG & $1977-06$ & 11,98 & 11,20 & 5,56 & 21,40 & 23,20 & 23,95 \\
AIN & $1977-06$ & 11,46 & 10,90 & 4,58 & 19,60 & 22,00 & 23,43 \\
JON & $1977-06$ & 13,23 & 12,40 & 5,43 & 22,80 & 24,70 & 24,69 \\
SAO & $1977-06$ & 13,44 & 12,80 & 6,97 & 24,40 & 26,50 & 27,78 \\
SAL & $1977-06$ & 13,51 & 12,98 & 5,91 & 23,20 & 24,89 & 25,16 \\
ISE & $1977-06$ & 10,55 & 10,50 & 3,80 & 16,68 & 18,40 & 18,77 \\
SOY & $1977-06$ & 13,07 & 12,60 & 5,29 & 21,80 & 23,60 & 24,27 \\
CRU & $1977-06$ & 13,26 & 12,80 & 5,43 & 22,10 & 23,90 & 24,57 \\
TRI & $1977-06$ & 13,18 & 12,70 & 5,61 & 22,28 & 24,00 & 24,58 \\
ARA & $1977-06$ & 14,36 & 13,80 & 5,86 & 23,90 & 25,75 & 26,73 \\
\hline
\end{tabular}

$5^{\circ} \mathrm{C}$, et par deux affluents froids majeurs : l'Arve dont les effets sont sensibles jusqu'à Bugey, et l'Isère jusqu'à Tricastin. La Saône, plus froide en hiver dans des débits élevés et plus chaude en été dans des débits faibles, marque plus légèrement la thermie en augmentant légèrement l'amplitude du cycle annuel à l'aval.

Pour les statistiques indicatives de températures chaudes comme le quantile $99 \%$ ou la moyenne du mois le plus chaud, on observe que la valeur la plus élevée est trouvée sur la Saône, et que la température de sortie du Léman avoisine celle observée à Saint Alban et Tricastin, signe probable que dans ces conditions rares, le Rhône approche l'équilibre avec les conditions hydroclimatiques caniculaires (Edinger et al., 1968; Caissie et al., 2005). En canicule, des conditions thermiques similaires prévalent sur tout le bassin du Rhône, hormis sur les affluents ayant une composante nivale ou glaciaire forte (Brown \& Hannah, 2008), l'apport d'eau froide modifiant sensiblement la température.

\subsection{Analyse des régimes thermiques du Rhône et de ses principaux affluents}

Le régime thermique permet de caractériser les températures de l'eau en fonction du jour ou du mois de l'année. Il caractérise le pourcentage de chance de dépasser une valeur donnée un jour donné. Pour limiter l'effet d'échantillonnage lié au «faible» nombre d'années de mesures, les probabilités trouvées chaque jour sont lissées sous l'hypothèse qu'elles varient très peu d'un jour à l'autre : la température dépassée $50 \%$ des années le 10 juillet est nécessairement très proche de celle dépassée $50 \%$ des années le 9 ou le 11 juillet... 
T en ${ }^{\circ} \mathrm{C}$

Evolution longitudinale des températures de l'eau sur le Rhône et ses affluents: 1977-2006

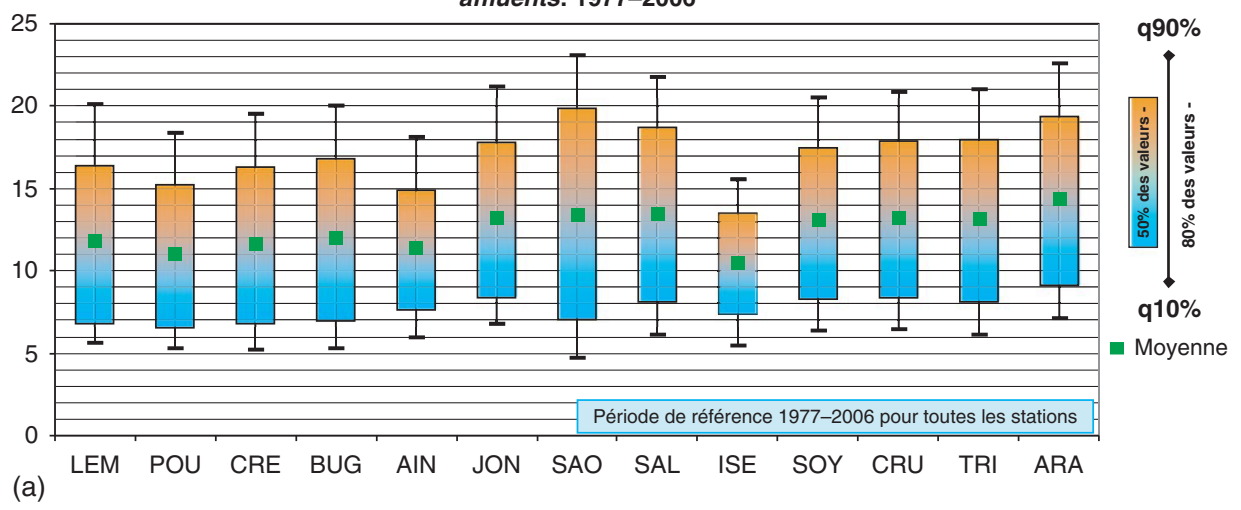

$\mathrm{T}$ en ${ }^{\circ} \mathrm{C}$

Evolution longitudinale des températures de l'eau sur le Rhône et ses affluents: 1997-2006

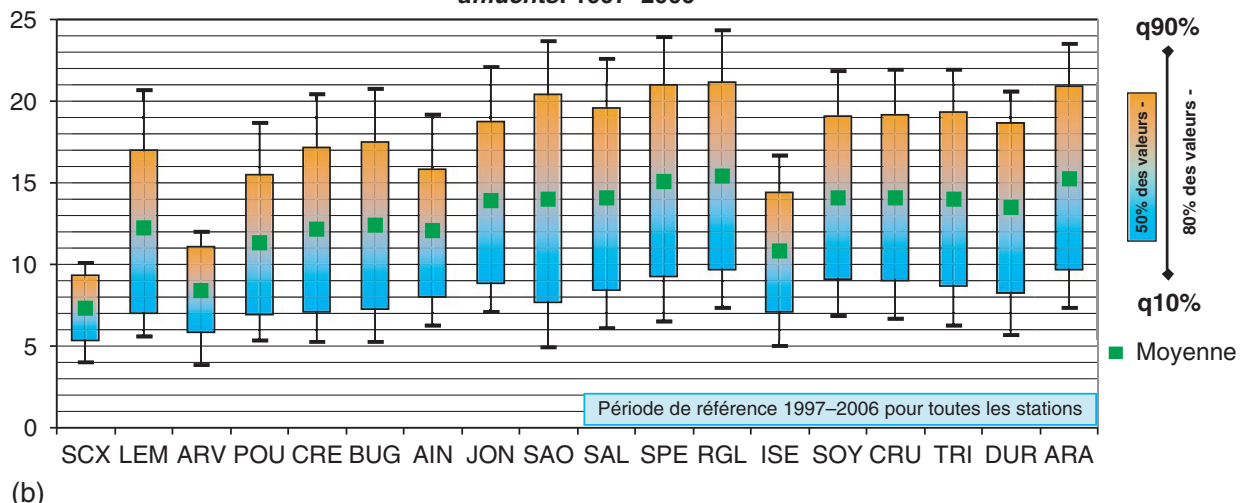

Fig. 3. Évolution longitudinale des températures observées sur le Rhône et ses principaux affluents (a) sur 1977-2006 et (b) sur 1997-2006.

Fig. 3. Longitudinal evolution of water temperature on the Rhône river and its main tributaries (a) from 1997 to 2006 and (b) from 1997 to 2006.

On peut utiliser différentes techniques de lissage; les séries de Fourier utilisées depuis le $\mathrm{XIX}^{\mathrm{e}}$ siècle (Plantamour, 1863) et reprises par de nombreux auteurs (Ward, 1963; Tasker, 1974; Salas, 1980; Steele, 1982) présentent de nombreux avantages à la fois statistiques et interprétatifs. Elles sont cependant moins bien adaptées que des moyennes glissantes pour traduire des évolutions de gestion à une date donnée.

Les séries de Fourier permettent de comparer simplement les moyennes, les amplitudes annuelles et les déphasages temporels (position du maximum et du minimum durant l'année), c'est pourquoi elles ont été retenues 


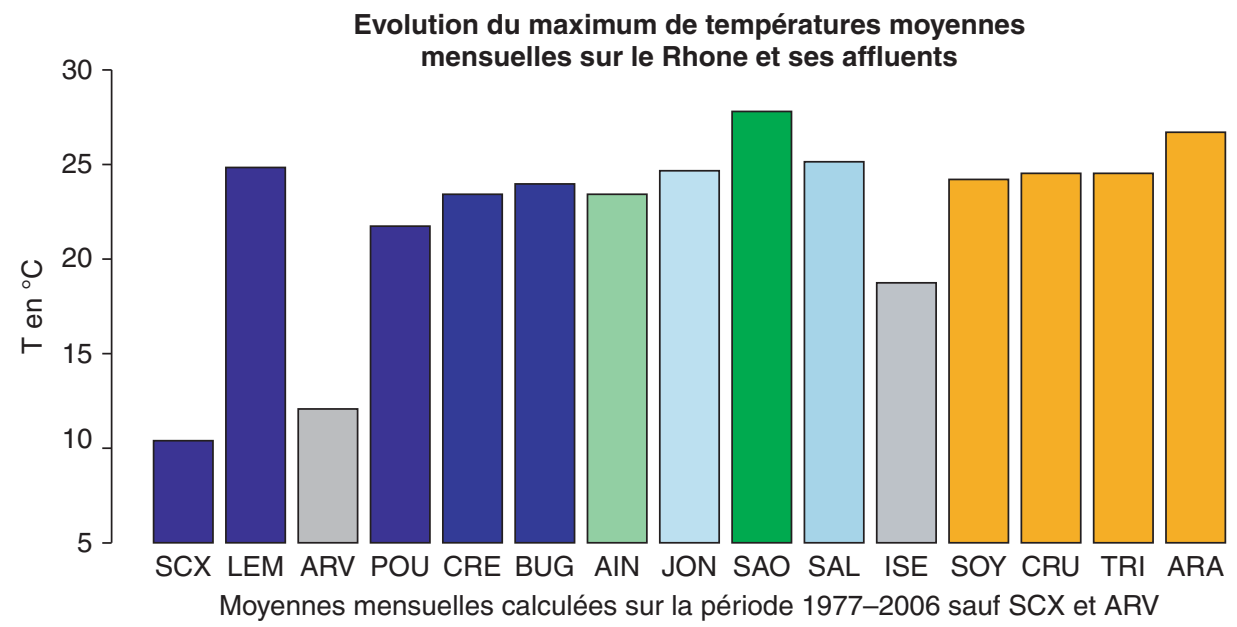

Fig. 4. Évolution longitudinale de la température moyenne du mois le plus chaud observée sur le Rhône et ses principaux affluents.

Fig. 4. Longitudinal evolution of the maximum monthly mean of water temperature on the Rhône river and its main tributaries.

dans cet article, contrairement à ce qui a été fait pour l'Étude Globale Rhône.

Une analyse a confirmé que deux harmoniques étaient nécessaires pour obtenir une bonne précision sur la courbe lissée, notamment pour certaines rivières comme la Saône. Ces séries de Fourier ont été utilisées pour déterminer des métriques biologiques soit directement, soit au travers des dates de passages de certains seuils thermiques (Poirel et al., 2001).

Pour chaque série chronologique, on peut également obtenir un faisceau de courbes quantifiant la probabilité de dépasser une certaine valeur de température de l'eau un jour donné. Sur la figure 5, les différentes années ont été tracées sous forme d'un chevelu et le régime lissé caractéristique de la station a été superposé pour des probabilités de dépassement de 0,$05 ; 0,10 ; 0,25 ; 0,50 ; 0,75$; 0,90 et 0,95 .
Ce type de graphique permet de situer très rapidement une température donnée par rapport à celles observées aux mêmes dates, les autres années : par exemple, les deux étés caniculaires de 2003 et 2006 avec des températures à plus de $26^{\circ} \mathrm{C}$ sortent nettement du faisceau de courbes...

Les figures 6,7 et 8 illustrent un autre usage de ces séries de Fourier : elles permettent d'obtenir une vision à la fois spatiale et temporelle sur une statistique donnée (ici la moyenne) ainsi que des comparaisons interstations.

On distingue très nettement les séries glacio-nivales du Rhône amont et de l'Arve, ainsi que la série nivale de l'Isère avec une amplitude annuelle nettement plus faible. Sur l'Isère et l'Arve, les débits estivaux marqués par l'apport important d'eaux froides issues de la fonte nivale et glaciaire induisent un régime thermique très caractéristique. 


\section{SAL - Régime lissé par série de Fourier}

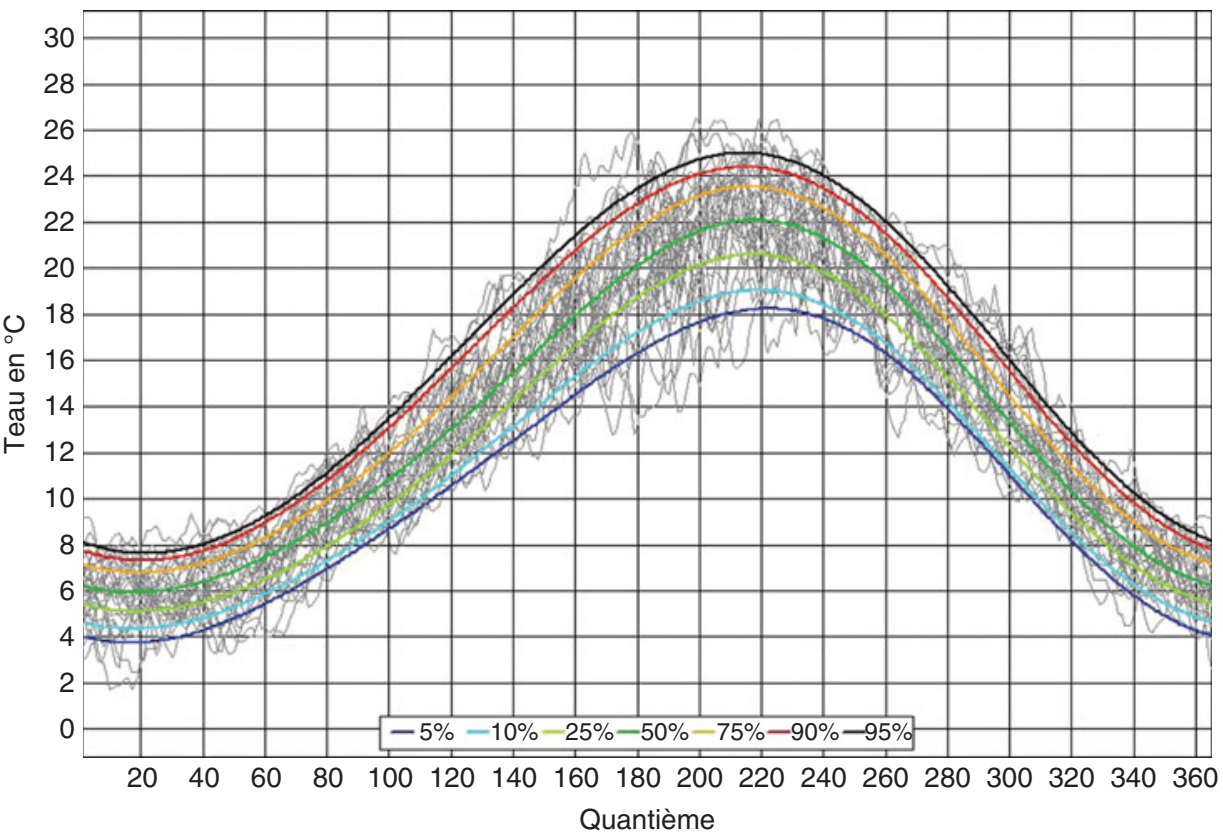

Fig. 5. Trente années de température journalière et régime lissé associé à la station de Saint Alban (SAL).

Fig. 5. Thirty years of water temperature measurement and thermal regime at Saint Alban sampling site (SAL).

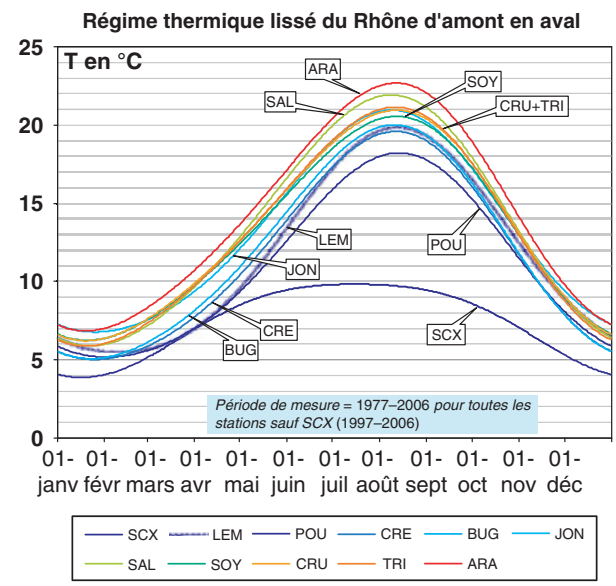

Régime thermique lissé de quelques affluents du Rhône

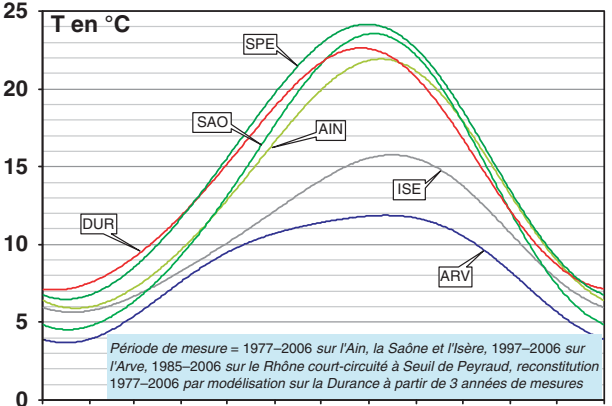

01- 01- 01- 01- 01- 01- 01- 01- 01- 01- 01- 01janv févr mars avr mai juin juil août sept oct nov déc

- ARV — ISE — AIN — SAO — DUR — SPE

Fig. 6 et 7. Régime thermique moyen du Rhône et de ses principaux affluents.

Fig. 6 and 7. Thermal regime of the Rhône river and its main tributaries. 


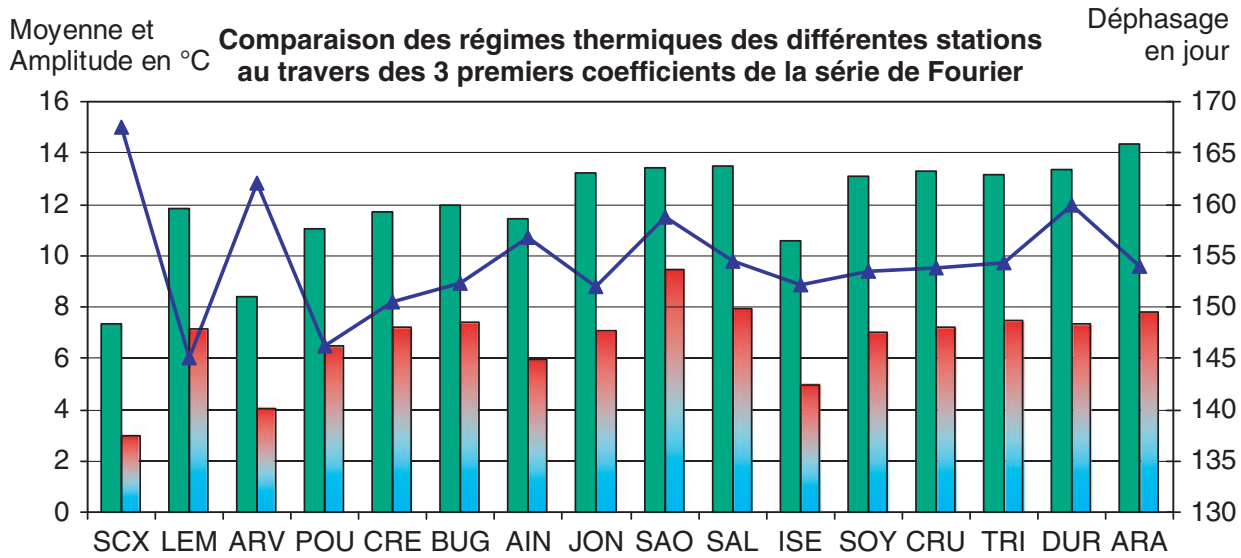

$\square$ Moyenne $\square$ Amplitude $\rightarrow$ Déphasage

Fig. 8. Évolution longitudinale des trois principaux coefficients de la série de Fourier (moyenne, amplitude et déphasage).

Fig. 8. Longitudinal evolution of the three main Fourier coefficients (mean, amplitude and phase).

Toutes les autres séries présentent une amplitude annuelle plus marquée et beaucoup plus en relation avec celle des températures de l'air.

Les maxima thermiques de ces courbes sont atteints pour la Saône et le Vieux-Rhône qui apparaissent proches de l'équilibre avec les conditions hydroclimatiques. Pour l'Isère et l'Ain, on peut supposer que l'influence des grands barrages (Vouglans et Monteynard) se traduit par une inertie plus élevée et donc des valeurs minimales beaucoup plus élevées (Webb et Walling, 1997).

\section{5 ÉVOLUTION DES TEMPÉRATURES DE L'EAU}

\subsection{Estimation des évolutions temporelles entre 1977 et 2006}

L'intérêt des longues séries temporelles est, bien sûr, de pouvoir évaluer des tendances ou des différences de comportement entre périodes. À l'heure où le changement climatique est largement étudié pour l'air, il était intéressant d'analyser les évolutions constatées sur les températures de l'eau (Pont, 2003; Daufresne et al., 2004) avec les nuances suivantes:

- trente années de mesure sont très insuffisantes pour définir le climat et il faudra donc bien se garder d'extrapoler les résultats obtenus;

- l'année 1977 a vu le lancement de nombreuses séries de mesures suite à la période caniculaire de 1976. A posteriori, au moins sur les températures de l'air, la décennie 1977-1987 s'est révélée plutôt fraîche. On démarre donc les observations dans une décennie froide pour les terminer dans la période chaude actuelle, d'où une tendance exagérée; 
- les températures de l'eau sont également très dépendantes d'autres facteurs comme le débit le vent, l'humidité (Smith, 1981; Mohseni \& Stefan, 1999; Benyahya et al., 2007). Par exemple, à des températures de l'air caniculaires correspondent en général des débits soutenus et très froids pour les rivières glacio-nivales (fontes des glaces) et des débits faibles et chauds pour des rivières de plaine, d'où la complexité des processus en jeu (Brown et al., 2006).

\section{2 Évolution des températures moyennes annuelles}

Hormis le Rhône à l'amont du Léman (série courte), toutes les séries présentent une évolution temporelle positive de leurs moyennes (Fig. 9). Cette évolution est plus marquée sur le Rhône aval que sur le Rhône amont. L'année 1987 semble faire une rupture avec les années suivantes nettement plus chaudes.

Hormis l'Arve (série courte), tous les affluents présentent une tendance à la hausse des températures de l'eau (Fig.10). Les rivières plus proches de l'équilibre avec les conditions atmosphériques (Ain et Saône) présentent les évolutions les plus marquées. L'Isère, l'Arve et le Rhône amont, semblent moins affectées ce qui est probablement à relier à la composante glacio-nivale.

Ces tendances sont assez comparables à ce qui a été observé ou prévu par différents auteurs sur d'autres rivières et fleuves (Langan et al., 2001; Webb \& Walling, 1992; Moatar \& Gailhard, 2006 ; Webb \& Nobilis, 2007).

\section{3 Évolution des températures moyennes décennales}

Le tableau III et la figure 11 montrent que, quelles que soient la rivière, la station et la décennie concernées, la moyenne de la décennie récente est toujours plus élevée que celle de la décennie plus ancienne...

Cette différence cumulée entre les années [1977-1986] et les années [1997-2006] est d'environ $1^{\circ} \mathrm{C}$ du Léman au Bugey ainsi que sur la Saône, elle atteint $1,5^{\circ} \mathrm{C}$ sur l'Ain et l'aval du Bugey, puis passe à $2^{\circ} \mathrm{C}$ environ sur l'aval de l'Isère.

\section{4 Évolution des températures moyennes par saison}

Outre l'analyse des températures moyennes annuelles et inter-annuelles, et dans une approche plus biologique, il est aussi intéressant d'analyser ces évolutions par saison (Fig. 12).

Pour tous les sites, on observe des évolutions très sensibles entre la décennie 1977-1986 et la dernière décennie notamment au printemps comme en été. Pour ces mêmes saisons, sur l'Ain, l'Isère et le Rhône à l'aval de l'Isère notamment, les valeurs moyennes de la dernière décennie sont celles rencontrées moins de $10 \%$ du temps dans la décennie 1977-1986.

\section{UNE SINGULARITÉ} RHODANIENNE HISTORIQUE : LES «GOUTTES FROIDES » DU LÉMAN

Le Rhône en aval immédiat du Léman présente un comportement spécifique par rapport aux autres grands 
Evolution temporelle des températures moyennes annuelles sur le Rhône

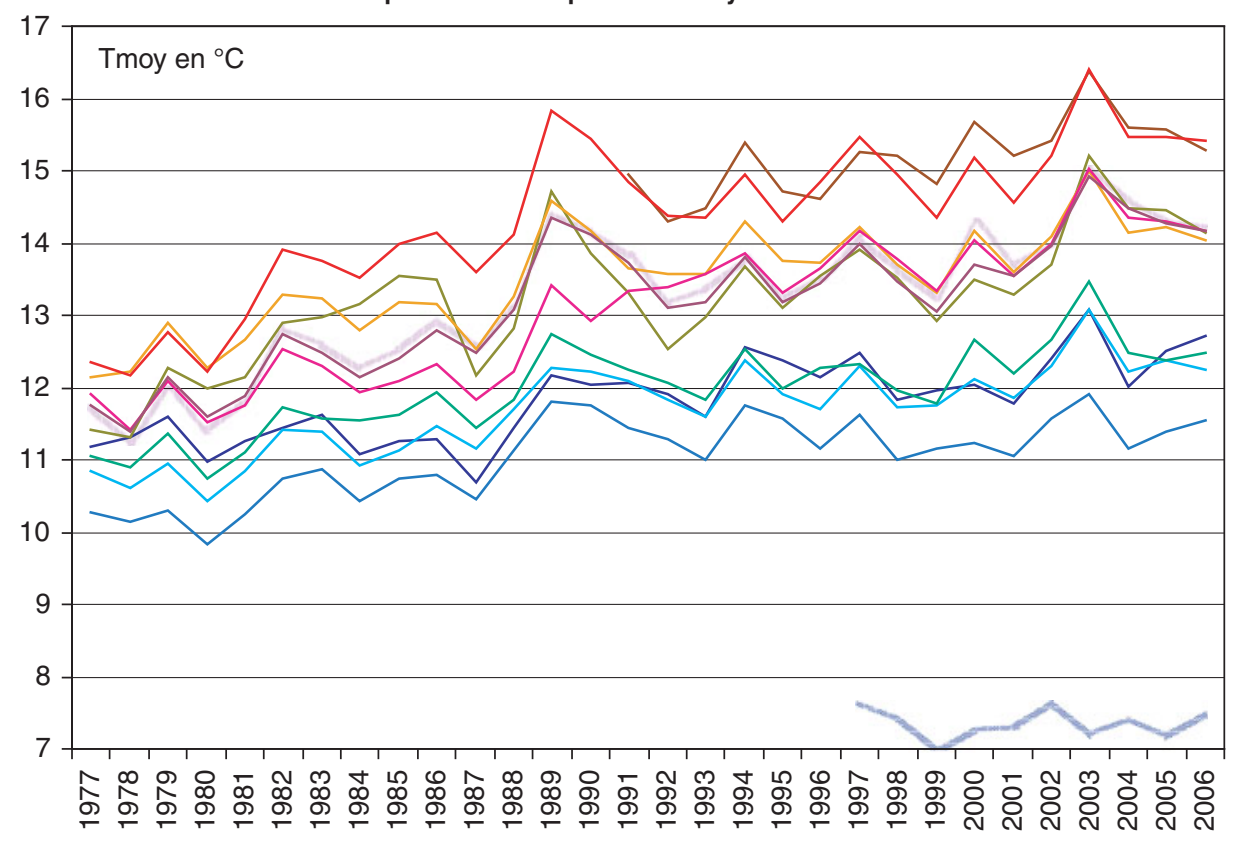

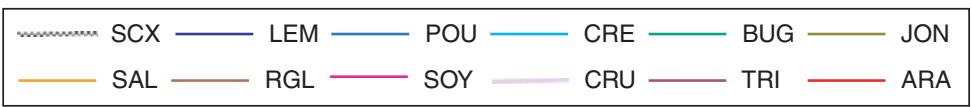

Fig. 9. Évolution des températures de l'eau moyennes annuelles sur le Rhône.

Fig. 9. Temporal evolution of annual mean water temperature on the Rhône river.

fleuves issus d'autres lacs européens. Depuis plus de 200 ans au moins, il existe des baisses de température rapides pouvant dépasser $10^{\circ} \mathrm{C}$ d'amplitude et durer deux ou trois jours, avec des gradients de baisse de plus de $1^{\circ} \mathrm{C} /$ heure. Ce comportement très particulier offre l'intérêt de tracer thermiquement les masses d'eaux jusqu'à la Méditerranée et il permet l'étude biologique des modifications thermiques brutales assez similaires à celles signalées en aval de certaines retenues hydroélectriques (Jones et Preece, 2002).
Ces apports d'eaux froides sont mentionnés dès le XVIII siècle par HB de Saussure (De Saussure, 1779) dans son voyage au travers des Alpes; ils apparaissent, à l'époque, aléatoires et d'origine inconnue, même si la température froide au fond des lacs est déjà connue par les premiers profils de température.

Dès le milieu du XIXe, ils sont mis en relation avec des courants de surface inverses dans le Léman (courants allant de Genève vers l'arrivée du Rhône); ces courants ou "ladières ", sont eux-mêmes favorisés par des 


\section{Evolution temporelle des températures moyennes annuelles} sur les affluents du Rhône et le Rhône court-circuité

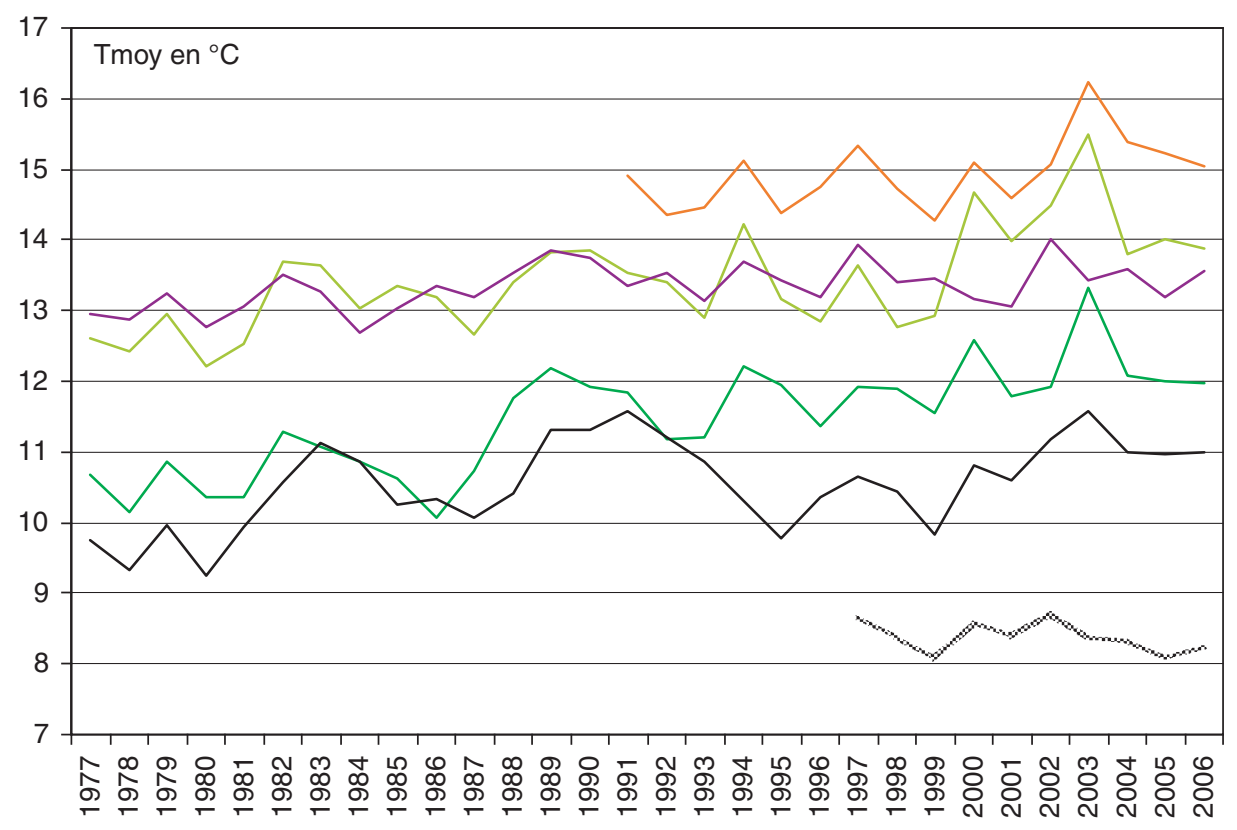

AIN $\longrightarrow$ SAO $\longrightarrow$ SPE $\longrightarrow$ ISE $\longrightarrow$ DUR

Fig. 10. Évolution des températures de l'eau moyennes annuelles sur les affluents du Rhône et sur le Rhône court-circuité.

Fig. 10. Annual mean water temperature evolution on the main tributaries of the Rhône river and on a by-passed section of the Rhône.

Tableau III. Températures moyennes par décennies et écarts entre les décennies successives.

Table III. Mean water temperature by decade and differences between successive decades.

\begin{tabular}{|c|cccccccccccccccccc|}
\hline moy/décennie & SCX & LEM & ARV & POU & CRE & BUG & AIN & JON & SAO & SAL & SPE & RGL & ISE & SOY & CRU & TRI & DUR & ARA \\
\hline $1977-1986$ & & 11.3 & & 10.4 & 11.0 & 11.4 & 10.6 & 12.5 & 13.0 & 12.8 & & & 10.1 & 12.0 & 12.1 & 12.1 & 13.1 & 13.2 \\
$1987-1996$ & & 11.9 & & 11.3 & 11.9 & 12.1 & 11.6 & 13.3 & 13.4 & 13.7 & 14.7 & 14.7 & 10.7 & 13.2 & 13.5 & 13.4 & 13.5 & 14.7 \\
$1997-2007$ & 7.4 & 12.3 & 8.4 & 11.4 & 12.2 & 12.4 & 12.1 & 13.9 & 14.0 & 14.0 & 15.1 & 15.4 & 10.8 & 14.1 & 14.1 & 14.0 & 13.5 & 15.2 \\
\hline \multicolumn{2}{|c|}{} & & & & & & & & & & & & & & \\
\hline Ecarts & SCX & LEM & ARV & POU & CRE & BUG & AIN & JON & SAO & SAL & SPE & RGL & ISE & SOY & CRU & TRI & DUR & ARA \\
\hline$[87-96]-[77-86]$ & 0.60 & & 0.90 & 0.89 & 0.78 & 1.00 & 0.76 & 0.42 & 0.93 & & & 0.58 & 1.16 & 1.38 & 1.32 & 0.39 & 1.49 \\
{$[97-06]-[87-96]$} & & 0.38 & & 0.03 & 0.31 & 0.30 & 0.47 & 0.63 & 0.58 & 0.34 & 0.44 & 0.70 & 0.08 & 0.92 & 0.59 & 0.51 & 0.02 & 0.58 \\
\hline
\end{tabular}

vents orientés Ouest-Est (Vallée, 1843). Seul le mécanisme de mise en place de la couche froide dans le
Léman, attribuée à des apports sous lacustres des glaciers alpins, est de nos jours invalidé... 


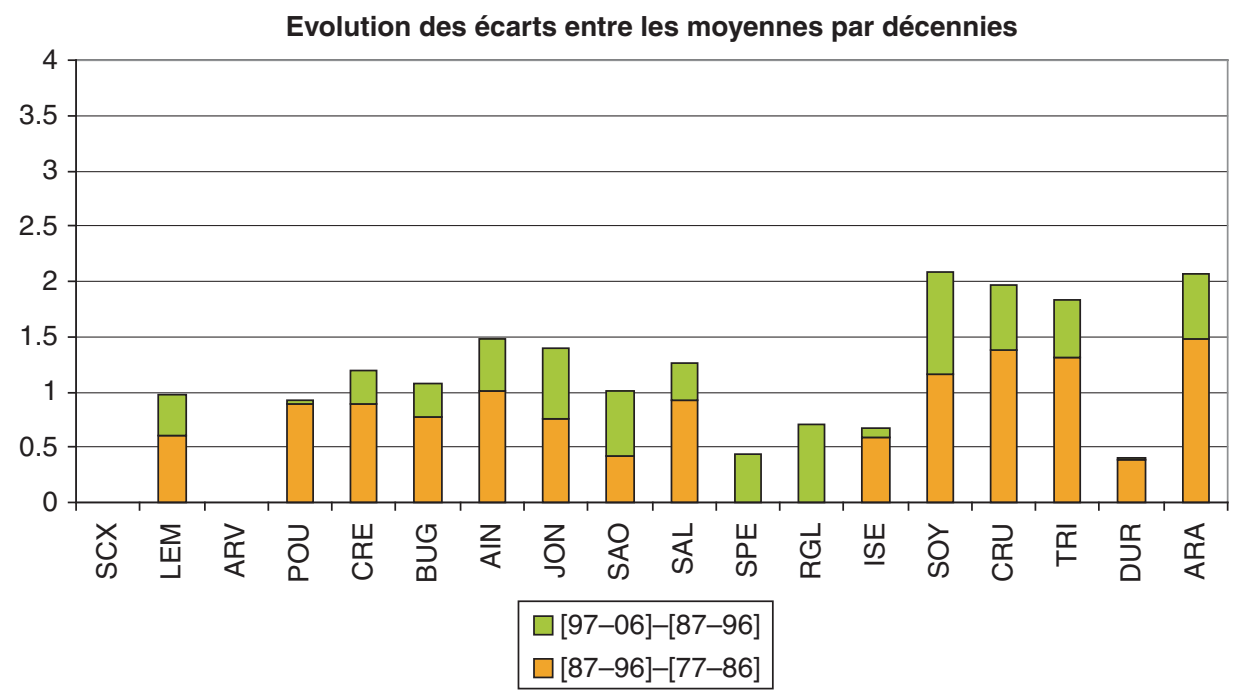

Fig. 11. Augmentation cumulée des températures moyennes de l'eau par décennies.

Fig. 11. Cumulative increase of mean water temperature by decades.

Le responsable de l'observatoire météorologique de Genève (Plantamour, 1863) indique au terme de 4 années de mesures journalières que, par vents du Sud, en été, on peut observer un abaissement de 6 à $10^{\circ} \mathrm{C}$ sur une journée, de même qu'en hiver par vent du Nord mais dans une moindre mesure.

Plus tard les travaux des limnologues (Forel, 1892; Delebecque, 1898) permettront de comprendre la formation de la thermocline et d'expliquer la formation de la couche profonde froide ainsi que la dynamique des courants lacustres (Hubert, 1971).

Au-delà de leur cause initiale, ces "accidents " thermiques majeurs se répercutent jusqu'à la Méditerranée en s'étalant et en s'atténuant, permettant ainsi de mettre en évidence les processus en jeu.
Les chutes de températures qui atteignent plus de $10^{\circ} \mathrm{C}$ en quelques heures et peuvent durer deux à trois jours (Fig. 13) pour des volumes d'eau de quelques dizaines de millions de $\mathrm{m}^{3}$. Leurs effets, progressivement atténués, se propagent jusqu'à la Méditerranée

Ils tracent ainsi les déplacements des masses d'eau tout au long du système fluvial et montrent

- que le Rhône, est très loin de l'équilibre avec les conditions hydrométéorologiques locales et ne correspond donc pas au concept de rivière de plaine;

- que les temps de transfert des masses d'eau (9 à 12 jours) sont très différents du temps de transfert des débits (moins de 24 heures) ce qui s'explique par l'artificialisation des débits du fleuve; 

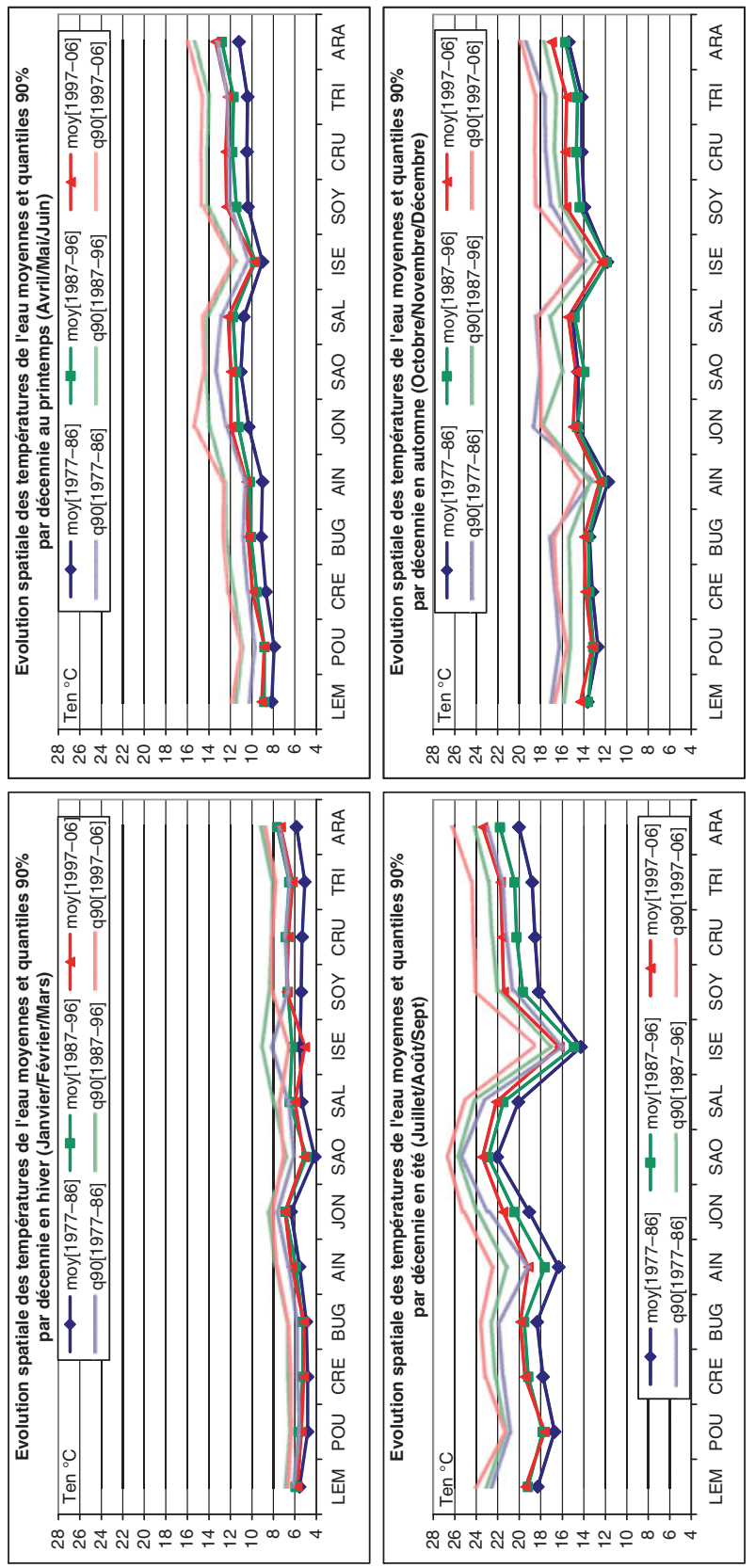

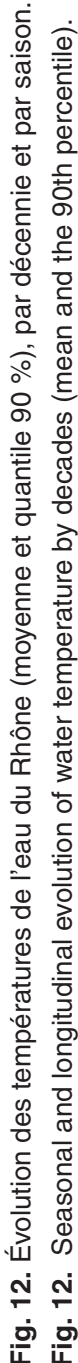


Transfert de la "goutte froide" de la fin Juin 2003

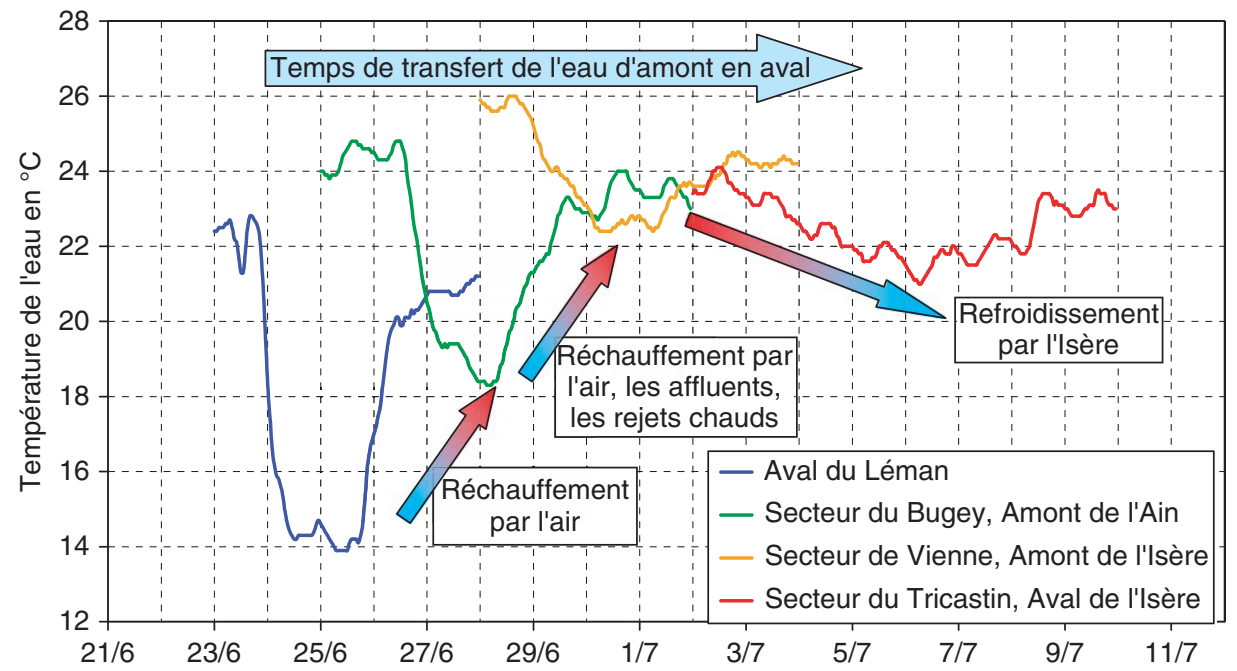

Fig. 13. Transfert vers l'aval d'une "goutte froide " hypolimnique du lac Léman à la Méditerranée.

Fig. 13. Downstream transfer of hypolimnic cold water, called "cold drop", from the Geneva Lake to the Mediterranean sea, at the end of June 2003.

\section{CONCLUSIONS}

Avec trente années de mesures horaires des températures de l'eau sur plus de quinze stations sur le Rhône et ses affluents, le bassin rhodanien apparaît aujourd'hui comme un des mieux connus sur le plan thermique. Ces mesures qui nécessitent un investissement matériel important et une validation des données au fil du temps sur la durée sont aujourd'hui riches d'enseignements, confirmant ainsi tout l'intérêt de stations patrimoniales de température de l'eau.

La première phase de l'étude thermique globale du Rhône, commanditée par l'Administration française et objet de cet article, a permis de faire un état de la thermie du Rhône. Elle a servi de base incontournable aux phases suivantes centrées sur la compréhension des processus thermiques et des effets biologiques.

Ces séries permettent donc de définir le régime thermique du fleuve avec une vision à la fois spatiale et temporelle. Elles confirment les tendances à la hausse observées sur d'autres bassins, que ce soit sur le Rhône lui-même ou sur ses affluents. Seules les stations dont le régime hydrologique est glacio-nival semblent épargnées par ces élévations, l'apport important d'eaux froides en été compensant probablement la hausse des températures de l'air.

Ces hausses de températures sont moins nettes en automne, mais elles sont très marquées au printemps et en été, où la valeur moyenne de la dernière décennie est très proche du 
quantile $90 \%$ de la décennie [19771986].

Enfin, ces séries mettent en évidence des phénomènes transitoires en été, liés à des apports hypolimniques du Léman sur des durées de un à trois jours. Ces « gouttes froides » se propagent tout au long du système jusqu'à la Méditerranée. Elles constituent d'excellents traceurs du déplacement des masses d'eau dans un système anthropisé et permettent une compréhension fine des processus en jeu.

Ces longues séries apparaissent donc aujourd'hui fondamentales pour la compréhension de la thermique du fleuve et constituent une base importante dans l'analyse des évolutions biologiques. Elles servent aussi à caler des modèles prévisionnels afin d'optimiser la gestion des rejets chauds des centrales thermiques (nucléaires ou classiques).

\section{RÉFÉRENCES \\ BIBLIOGRAPHIQUES}

Beitinger T.L. \& Bennett W.A., 2000. Quantification of the role of acclimation temperature in temperature tolerance of fishes. Environ. Biol. Fish. 58 : 277288.

Benyahya L. et al., 2007. Modeling of water temperatures based on stochastic approaches : case study of the Deschutes River. J. Environ. Engineer. Sci. 6(4) : 437-448.

Brown L.E. \& Hannah D.M., 2008. Spatial heterogeneity of water temperature across an alpine river basin. Hydrological Processes 22 (7), 954.

Brown L.E., Hannah D.M. \& Milner A.M., 2006. Hydroclimatological influences on water column and streambed thermal dynamics in an alpine river system. J. Hydrology 325 (1-4) : 1-20.

Caissie D., Satish, M.G. \& El-Jabi N., 2005. Predicting water temperatures using the equilibrium temperature concenpt with application on Miramichi River catchments (New Brunswick, Canada). Hydrological Processes 19 : 21372159.

Daufresne M. et al., 2004. Long-term changes within the invertebrate and fish communities of the Upper Rhône River : effects of climatic factors. Global Change Biology 10 (1) : 124-140.

Daufresne M. \& Boet P., 2007. Climate change impacts on structure and diversity of fish communities in rivers. Global Change Biology 13(12) : 24672478.

Delebecque A., 1898. Les Lacs Français, Paris : Chamerot et Renouard.

Eaton J.G. et al., 1995, A Field Informationbased System for Estimating Fish Temperature Tolerances. Fisheries 20 (4) : 10-18.

Ebersole J., Liss W. \& Frissel C., 2003. Thermal heterogeneity, stream channel morphology, and salmonid abundance in northeastern Oregon streams. Canad. J. Fish. Aquatic Sci. $60: 1266-1280$.

Edinger J.E., Duttweiler D.W. \& Geter J.C., 1968. The response of water temperatures to meteorological conditions. Water Resources Research 4(5) : 11371143.

Forel F.A., 1892. Le Léman, monographie limnologique, F Rouge Editeur, Lausanne.

Grenouillet G. et al., 2001. Large-scale synchrony and inter-annual variability in roach recruitment in the Rhône River: the relative role of climatic factors and density-dependent processes. Freshwater Biology 46 (1) : 11-26.

Hubert P., 1971. Étude par le Tritium de la dynamique des Eaux du Lac Léman. 
Thèse de l'Université Pierre et Marie Curie, 149 p.

Jones H.A. \& Preece R.M., 2002. The effect of Keepit Dam on the temperature regime of the Namoi River, Australia. River Research and Applications 18 (4) : 397-414.

Langan S. et al., 2001. Variation in river water temperatures in an upland stream over a 30-year period. The Science of the Total Environment 265 : 195-207.

Moatar F., 1997. Modélisation statistiques et déterministes des paramètres physico-chimique utilisés en surveillance des eaux de rivières : Application à la validation des séries de mesures en continu (cas de la Loire Moyenne). Thèse de l'Institut National Polytechnique de Grenoble, 290p.

Moatar F. \& Gailhard J., 2006. Water temperature behaviour in the River Loire since 1976 and 1881. Comptes Rendus Geosciences $338(5)$ : 319-328.

Mohseni O. \& Stefan H.G., 1999. Stream temperature/air temperature relationship : a physical interpretation. J. Hydrology 218 (3-4) : 128-141.

Plantamour E. 1863. Du Climat de Genève. Éditeur : Henri GEORG, Genève.

Poirel A., Carrel G. \& Olivier J.M., 2001. Régime thermique du Rhône et peuplements piscicoles. Dans Lyon Grands Fleuves 2001. Lyon.

Pont D., 2003. Conséquences potentielles du changement climatique sur les biocénoses aquatiques et riveraines françaises, GICC - Aquabio.

Salas J.D., 1980. Applied Modeling of Hydrologic Time Series, Water Resources Publication. de Saussure H.B., 1779. Voyage dans les Alpes, Neuchatel : Editeur - Louis FAUCHE-BOREL, 1803.

Smith K., 1981. The Prediction of River Water Temperatures. Hydrological Sciences Bulletin 26(1).

Steele T., 1982. A characterization of stream temperature in Pakistan using harmonic analysis. Hydrological Sciences journal 4 (12) : 451-467.

Tasker G.D. \& Burns A.W., 1974. Mathematical Generalization of Stream Temperature in Central New England. Journal of the American Water Resources Association 10 (6) : 1133-1142.

Vallee L., 1843. Du Rhône et du Lac de Genève. Des grands travaux à exécuter pour la Navigation du Léman à la Mer Librairie Scientifique Industrielle., Paris : L.Mathias.

Ward J., 1963. Annual variation of stream water temperature. Dans "Proceedings of the American Society of Civil Engineers". Journal of the Sanitary Engineering Division, p. 3710-3732.

Webb B.W. \& Walling D.E., 1997. Complex summer water temperature behaviour below a UK regulating reservoir. Regulated Rivers : Research \& Management 13 (5) : 463-477.

Webb B.W. \& Nobilis F., 2007. Long-term changes in river temperature and the influence of climatic and hydrological factors. Hydrological Sciences journal 52 (1) : 74-85.

Webb B. \& Walling D., 1992. Long term water temperature behaviour and trends in a Devon, UK, river system. Hydrological sciences journal 37 (6) : 567-580.

Westhoff M.C. et al., 2007. A distributed stream temperature model using high resolution temperature observations. Hydrology and Earth System Sciences $11: 1469-1480$. 
